\title{
A NON-LINEAR DIFFERENCE EQUATION WITH TWO PARAMETERS. II
}

\author{
A. BROWN ${ }^{1}$ \\ (Received 27 November 1984)
}

\begin{abstract}
The paper discusses solutions of period 4 for the difference equation

$$
y_{n+1}=2 k /\left\{1+\left(y_{n}-m\right)^{2}\right\} \text {, }
$$

where $k$ and $m$ are real parameters, with $k>0$. For given values of $k$ and $m$ there are at most three solutions with period 4 and equations are set up to determine the elements of these solutions and the stability of each solution. Only real solutions are considered. The procedure that is used to find these solutions allows unstable solutions to be identified as well as stable solutions.

In a previous paper, solutions of period 2 and period 3 were examined for this equation and there was evidence of anomalous behaviour in the way the stability intervals occurred. Some preliminary information about solutions of period 4 was mentioned in the discussion. The present paper provides more complete results, which confirm the anomalous behaviour and give a better idea of how the stability criterion changes for different families of solutions. These results are used to indicate the variety of behaviour that can be found for one-parameter systems by imposing suitable conditions on $m$ and $k$.
\end{abstract}

\section{Introduction}

In a previous paper [2], which will be cited as Paper I, the motivation for studying the equation

$$
y_{n+1}=F\left(y_{n}\right)=2 k /\left\{1+\left(y_{n}-m\right)^{2}\right\}
$$

was set out. It arose from an iteration formula for solving a cubic equation [4], and equation (1.1), with $m$ real and $k>0$, was seen to be a standardised form of the general problem. From that point of view, the main concern was to ensure

\footnotetext{
${ }^{1}$ Department of Theoretical Physics, Research School of Physical Sciences, Australian National University, G.P.O. Box 4, Canberra, A.C.T. 2601.

(c) Copyright Australian Mathematical Society 1985, Serial-fee code 0334-2700/85
} 
that the iteration procedure converged quickly, and an adequate method was developed in Section 7 of Paper I. At the same time it was of interest to have information about periodic solutions of this two-parameter difference equation, since most of the information for problems of this kind is for one-parameter equations [3], although more recently two-parameter equations have attracted attention in the literature $[1,5,6]$.

Solutions with minimum period 2 ( $C 2$ solutions) and with minimum period 3 (C3 solutions) were studied in Paper I, and Table 2 of that paper gives critical values of $k$ for a number of values of $m$. For a given value of $m, C 2$ solutions occur for $k>k^{*}$, where

$$
k^{*}=\left(1+m^{2}\right)\left\{-m+\sqrt{ }\left(1+m^{2}\right)\right\},
$$

and there is a single $C 2$ solution for each $k>k^{*}$. For $m<1$, all the $C 2$ solutions are stable, but for $m>1$ the $C 2$ solutions are stable for $k^{*}<k<k_{5}$, unstable for $k_{5}<k<k_{6}$, and stable again for $k>k_{6}$, where

$$
k_{5}=\left(1+m^{2}\right)\left\{m-\sqrt{ }\left(m^{2}-1\right)\right\}, \quad k_{6}=\left(1+m^{2}\right)\left\{m+\sqrt{ }\left(m^{2}-1\right)\right\} .
$$

When $k=k_{5}$ or $k=k_{6}$, the solution is on the border-line for stability, with the stability criterion, $S_{2}$, equal to -1 . When $m=1$, almost all the $C 2$ solutions are stable. In this case, $k_{5}=k_{6}=2$ and there is a $C 2$ solution with $S_{2}=-1$ when $k=2$. For all other admissible values of $k$, the $C 2$ solution has $-1<S_{2}<1$.

It turns out that the $C 4$ solutions (solutions with minimum period 4 ) occur for $m>1$ and $k_{5}<k<k_{6}$, that is, in the region where the $C 2$ solutions are unstable. For $m$ slightly greater than 1 there is only one $C 4$ solution for each admissible $k$ and these solutions are stable. This is the situation up to about $m_{1}=1.2$. (More precise values for $m_{1}$ and other critical values are given later.) The next critical value, $m_{2}$, is roughly 2.31 and for $m_{1}<m<m_{2}$, there is a single $C 4$ solution for each $k$, with two sub-intervals for $k$ in which the solutions are stable. One of these has $k_{5}$ as its lower limit while the other has $k_{6}$ as its upper limit. For $m>m_{2}$, there are three $C 4$ solutions for some values of $k$, and it is possible to have two more sub-intervals for $k$ where stable $C 4$ solutions occur. In Paper I it was noted that for $m=3$, superstable $C 4$ solutions could be identified for four different values of $k$, and in fact each of these superstable solutions occurs in a separate "window of stability" whose extent is determined in the course of this paper. This type of behaviour occurs for $m>m_{3}$, where $m_{3}$ is approximately 2.33. For $m_{2}<m<m_{3}$, there is an intermediate situation where stable $C 4$ solutions occur in three discrete $k$-intervals. Essentially these sub-divisions arise because the stability criterion for the relevant solutions goes from +1 to a minimum value, and then back to +1 (as $k$ increases, for a given value of $m$ ). If the minimum is less than -1 , two intervals of stability occur; otherwise there is 
a single interval of stability. Note that counting superstable solutions can be misleading. The number of superstable solutions in a stability interval can vary from 0 to 2, depending on the level of the minimum.

If we take $b_{1}, b_{2}, b_{3}, b_{4}$ to be the elements of a $C 4$ solution, then it is a matter of choice which element we label as $b_{1}$ and it is better initially to use a symmetric function of the $b_{i}$ such as the sum of the four elements, $\alpha$. Section 2 sets up various equations involving $\alpha$ and other symmetrical functions and in Section 3 these equations are combined to give a cubic equation for $\alpha$ (for given values of $m$ and $k$ ). Once $\alpha$ has been determined, other symmetrical functions follow and we can evaluate the elements of the solution and the stability criterion (Section 4). Some details of the numerical calculations are given in Section 5. It was found that the usual method of solution broke down for certain values of $m$ and $k$, where the equation for $\alpha$ has a double root, and this situation is considered in Section 6, together with some other special cases.

In Section 7, the results are re-examined in a different way. In discussing equation (1.1) we need information about the solutions for a two-dimensional array of points and this information was built up by seeing how the solutions changed along lines $m=$ constant. In effect, this gives solutions for a family of one-parameter problems, with $k$ as the parameter for each particular value of $m$. However we can also look at other families of one-parameter problems by, say, specifying $k$ as a function of $m$. Some examples are given in Section 7 and this brings out a greater variety of behaviour then before. It is clear that the "period-doubling path to chaos" is only one pattern out of a number of possibilities for one-parameter problems.

\section{Preliminary equations}

If equation (1.1) is iterated four times, to obtain $y_{n+4}$ as a function of $y_{n}$, equating $y_{n+4}$ to $y_{n}$ gives a polynomial equation of degree 17 . The roots of this equation include the elements of the $C 4$ solutions but they also include the $C 2$ solution and the equilibrium solutions, since $y_{n+4}=y_{n}$ holds for these degenerate cases also. The degenerate cases account for a factor of degree 5 in the polynomial equation and the remaining factor, of degree 12 , must provide the elements of the $C 4$ solutions. If $\left(b_{1}, b_{2}, b_{3}, b_{4}\right)$ is a typical $C 4$ solution, then

$$
h\left(y_{n}\right)=\left(y_{n}-b_{1}\right)\left(y_{n}-b_{2}\right)\left(y_{n}+b_{3}\right)\left(y_{n}-b_{4}\right)
$$

contributes a factor of degree 4 to the polynomial equation. We see that at most there can be three factors of this type in the polynomial of degree 12 , so at most there should be three $C 4$ solutions for each pair $(m, k)$. 
For a $C 4$ solution the elements $b$, must be positive and distinct, with

$$
2 k=\left(1+m^{2}\right) b_{2}-2 m b_{1} b_{2}+b_{1}^{2} b_{2}
$$

and with three similar equations obtained by cyclic permutation of the subscripts. Since it does not matter which element we take as $b_{1}$, it is convenient to work with functions of the $b_{i}$ which have cyclic symmetry, for example

$$
\sum b_{1}^{2} b_{2}=b_{1}^{2} b_{2}+b_{2}^{2} b_{3}+b_{3}^{2} b_{4}+b_{4}^{2} b_{1},
$$

where $\Sigma$ is used for cyclic summation over the subscripts $1,2,3$ and 4 . In particular we can use

$$
\begin{aligned}
& \alpha=\sum b_{1}, \quad \beta_{1}=\sum b_{1} b_{2}, \quad \beta_{2}=b_{1} b_{3}+b_{2} b_{4}, \\
& \gamma=\sum b_{1} b_{2} b_{3}, \quad \delta=b_{1} b_{2} b_{3} b_{4} .
\end{aligned}
$$

With this notation,

$$
h(y)=y^{4}-\alpha y^{3}+\beta y^{2}-\gamma y+\delta,
$$

where $\beta=\beta_{1}+\beta_{2}$, and if we can determine $\alpha, \beta_{1}, \beta_{2}, \gamma, \delta$ the elements $b_{1}$ are the roots of $h(y)=0$. Our procedure is to express $\gamma$ and $\delta$ in terms of $\alpha, \beta_{1}$ and $\beta_{2}$, then $\beta_{1}$ and $\beta_{2}$ in terms of $\alpha$ and finally to establish an equation for $\alpha$ (given $m$ and $k$ ). As noted in Section 1, the equation for $\alpha$ turns out to be a cubic, in line with our expectation that there are at most three $C 4$ solutions for any pair $(m, k)$.

A number of equations can be obtained directly from equation (2.2) and the three similar equations. Adding the equations gives

$$
\left(1+m^{2}\right) \alpha-2 m \beta_{1}+\sum b_{1}^{2} b_{2}=8 k .
$$

Multiplying equation (2.2) by $b_{3}$ and summing cyclically gives

$$
\left(1+m^{2}\right) \beta_{1}-2 m \gamma+\sum b_{1}^{2} b_{2} b_{3}=2 k \alpha
$$

and in the same way we can multiply equation (2.2) by $b_{4}, b_{2}, b_{3} b_{4}, b_{2} b_{3} b_{4}, b_{3} b_{4}^{2}$, $b_{4}^{2}-b_{2} b_{3}$ and then sum cyclically in each case. This gives

$$
\begin{aligned}
& 2\left(1+m^{2}\right) \beta_{2}-2 m \gamma+\sum b_{1} b_{2}^{2} b_{3}=2 k \alpha, \\
& \left(1+m^{2}\right) \sum b_{1}^{2}-2 m \sum b_{1} b_{2}^{2}+\sum b_{1}^{2} b_{2}^{2}=2 k \alpha, \\
& \left(1+m^{2}\right) \gamma-8 m \delta+\alpha \delta=2 k \beta_{1}, \\
& \left(1+m^{2}\right) \sum b_{1}^{2} b_{2} b_{3}-2 m \alpha \delta+\beta_{1} \delta=2 k \gamma, \\
& \left(1+m^{2}\right) \sum b_{1} b_{2} b_{3}^{2}-2 m \alpha \delta+\beta_{1} \delta=2 k \sum b_{1} b_{2}^{2}, \\
& \left(1+m^{2}\right) \sum\left(b_{1}^{2} b_{3}-b_{2}^{2} b_{3}\right)-2 m \sum\left(b_{1}^{2} b_{2} b_{3}-b_{1} b_{2}^{2} b_{3}\right) \\
& =2 k\left(-\beta_{1}+\sum b_{1}^{2}\right) .
\end{aligned}
$$


We can also make use of identities such as

$$
\begin{aligned}
& \sum b_{1}^{2}=\alpha^{2}-2 \beta, \\
& \sum\left(b_{1}^{2} b_{2}+b_{1} b_{2}^{2}\right)=\alpha \beta_{1}-2 \gamma, \\
& \sum b_{1}^{2} b_{3}=\alpha \beta_{2}-\gamma=\sum b_{1} b_{3}^{2}, \\
& \sum b_{1}^{2} b_{2}^{2}+2 \sum b_{1} b_{2}^{2} b_{3}=\beta_{1}^{2}-4 \delta, \\
& \sum b_{1} b_{2}^{2} b_{3}=\alpha \gamma-4 \delta-\beta_{1} \beta_{2}, \\
& \sum\left(b_{1}^{2} b_{2} b_{3}+b_{1} b_{2} b_{3}^{2}\right)=\beta_{1} \beta_{2}, \\
& \sum\left(b_{1}^{2} b_{2}^{2} b_{3}+b_{1} b_{2}^{2} b_{3}^{2}\right)=\beta_{1} \gamma-2 \alpha \delta .
\end{aligned}
$$

Another way of using (2.2) is to multiply by $b_{4}$ and to subtract the corresponding equation with the subscripts moved forward (cyclically) by two. This gives

$$
2 k\left(b_{4}-b_{2}\right)=b_{2} b_{4}\left(b_{3}-b_{1}\right)\left(2 m-b_{1}-b_{3}\right) \text {. }
$$

Similarly,

$$
2 k\left(b_{1}-b_{3}\right)=b_{3} b_{1}\left(b_{4}-b_{2}\right)\left(2 m-b_{2}-b_{4}\right),
$$

and we can multiply the two equations together, cancel out a non-zero factor $\left(b_{1}-b_{3}\right)\left(b_{2}-b_{4}\right)$ and end with

$$
-4 k^{2}=\delta\left(4 m^{2}-2 m \alpha+\beta_{1}\right) .
$$

\section{Combination of equations in Section 2}

In equation (2.11), we can substitute for $\sum b_{1}^{2} b_{3}, \Sigma b_{2}^{2} b_{3}, \Sigma b_{1}^{2} b_{2} b_{3}, \Sigma b_{1} b_{2}^{2} b_{3}$ and $\Sigma b_{1}^{2}$ from equations (2.14), (2.4), (2.5), (2.6) and (2.12). This gives

$$
\begin{aligned}
\gamma\left(1+m^{2}\right)= & 6 k \beta_{1}+\left\{(\alpha-4 m)\left(1+m^{2}\right)+4 k\right\} \beta_{2} \\
& +\alpha\left(1+m^{2}\right)^{2}-8 k\left(1+m^{2}\right)-2 k \alpha^{2},
\end{aligned}
$$

or if we put $2 k=p\left(1+m^{2}\right)$

$$
\gamma=3 p \beta_{1}+(\alpha-4 m+2 p) \beta_{2}+(\alpha-4 p)\left(1+m^{2}\right)-p \alpha^{2} .
$$

In the same way, we can eliminate $\Sigma b_{1}^{2}, \Sigma b_{1}^{2} b_{2}, \Sigma b_{1} b_{2}^{2}, \Sigma b_{1}^{2} b_{2}^{2}$ and $\Sigma b_{1} b_{2}^{2} b_{3}$ between equations (2.4), (2.6), (2.7), (2.12), (2.13) and (2.15). This gives

$$
\begin{aligned}
\beta_{1}^{2}= & 4 \delta+\left(2+2 m \alpha-2 m^{2}\right) \beta_{1}-\left(2+2 m^{2}\right) \beta_{2} \\
& +\left(1+m^{2}\right)\left\{p(3 \alpha-8 m)+2 m \alpha-\alpha^{2}\right\} .
\end{aligned}
$$

A similar equation, obtained by eliminating $\sum b_{1} b_{2}^{2} b_{3}$ between equations (2.6) and (2.16), is

$$
\beta_{1} \beta_{2}=(\alpha-2 m) \gamma-4 \delta+\left(1+m^{2}\right)\left(2 \beta_{2}-p \alpha\right)
$$


The summations on the left-hand side of equation (2.18) do not occur in any of the other equations, but we can write

$$
\begin{aligned}
\sum b_{1}^{2} b_{2}^{2} b_{3} & =\sum\left\{\left(b_{2} b_{3}\right)\left(b_{1}^{2} b_{2}\right)\right\}=\sum b_{2} b_{3}\left\{2 k-\left(1+m^{2}\right) b_{2}+2 m b_{1} b_{2}\right\} \\
& =2 k \beta_{1}-\left(1+m^{2}\right) \sum b_{1}^{2} b_{2}+2 m \sum b_{1} b_{2}^{2} b_{3}
\end{aligned}
$$

and the summations on the right-hand side are available from equations (2.4) and (2.0). In the same way,

$$
\begin{aligned}
\sum b_{1} b_{2}^{2} b_{3}^{2} & =\sum\left\{\left(b_{1} b_{3}\right)\left(b_{2}^{2} b_{3}\right)\right\}=\sum b_{1} b_{3}\left\{2 k-\left(1+m^{2}\right) b_{3}+2 m b_{2} b_{3}\right\} \\
& =4 k \beta_{2}-\left(1+m^{2}\right) \sum b_{1} b_{3}^{2}+2 m \sum b_{1} b_{2} b_{3}^{2} .
\end{aligned}
$$

Now $\sum b_{1} b_{3}^{2}$ can be replaced from equation (2.14) and we can combine equations (2.17), (2.5) and (3.4) to give

$$
\sum b_{1} b_{2} b_{3}^{2}=(\alpha-4 m) \gamma-4 \delta+\left(1+m^{2}\right)\left(\beta_{1}+2 \beta_{2}-2 p \alpha\right) \text {. }
$$

With this information available for the two summations in equation (2.18) we can make use of this equation and obtain

$$
\begin{aligned}
\left(4 m^{2}-\right. & \left.2 m \alpha+\beta_{1}\right) \gamma \\
= & \alpha \delta+\left(1+m^{2}\right)\left\{2 p \beta_{1}+(2 p-\alpha) \beta_{2}-2 m p \alpha\right\}+(\alpha-4 p)\left(1+m^{2}\right)^{2} .
\end{aligned}
$$

This is after using equation (2.8) to simplify the terms involving $\delta$.

If we compare equations (2.21) and (3.6), we note that the co-factor of $\delta$ in the first of these equations is the same as the co-factor of $\gamma$ in the second. This suggests that we combine them, using equation (2.8) in the process. From equation (2.8),

$$
\begin{gathered}
\left(4 m^{2}-2 m \alpha+\beta_{1}\right)\left\{\left(1+m^{2}\right) \gamma+\delta(\alpha-8 m)\right\} \\
=\left(4 m^{2}-2 m \alpha+\beta_{1}\right)\left\{p\left(1+m^{2}\right) \beta_{1}\right\} \\
=\left(1+m^{2}\right)\left\{p\left(4 m^{2}-2 m \alpha\right) \beta_{1}+p \beta_{1}^{2}\right\} .
\end{gathered}
$$

If we use equations (3.6) and (2.21) on the left-hand side and equation (3.3) to replace $\beta_{1}^{2}$ on the right-hand side, the resulting equation is

$$
(\alpha-4 p)\left(1+m^{2}\right)\left\{\delta-\left(1+m^{2}\right)\left(\beta_{2}-1-m^{2}-p \alpha+4 m p\right)\right\}=0 .
$$

Hence, provided $\alpha \neq 4 p$, we come out with

$$
\delta=\left(1+m^{2}\right)\left\{\beta_{2}-1-m^{2}-p(\alpha-4 m)\right\} .
$$

Strictly we should add another proviso, that $\alpha \neq 8 \mathrm{~m}$, since we multiplied equation (2.21) by $\alpha-8 m$ in the argument above.

If we substitute for $\gamma$ and $\delta$ in equation (2.8), using equations (3.2) and (3.9), we can cancel a common factor $2+2 m^{2}$ and get

$$
p \beta_{1}+(\alpha+p-6 m) \beta_{2}=p\left(\alpha^{2}-6 m \alpha+2+18 m^{2}\right)-4 m\left(1+m^{2}\right) .
$$


Similarly, equations (3.3) and (3.4) can be rewritten as

$$
\begin{aligned}
\beta_{1}^{2}= & \left(2+2 m \alpha-2 m^{2}\right) \beta_{1}+2\left(1+m^{2}\right) \beta_{2} \\
& +\left(1+m^{2}\right)\left\{p(8 m-\alpha)+2 m \alpha-\alpha^{2}\right\}-4\left(1+m^{2}\right)^{2}, \\
\beta_{1} \beta_{2}= & (3 \alpha-6 m) p \beta_{1}+\left\{(2 \alpha-4 m) p+\alpha^{2}-6 m \alpha+6 m^{2}-2\right\} \beta_{2} \\
+ & p\left\{-\alpha^{3}+2 m \alpha^{2}-(\alpha+8 m)\left(1+m^{2}\right)\right\} \\
+ & \left(1+m^{2}\right)\left(\alpha^{2}-2 m \alpha+4+4 m^{2}\right) .
\end{aligned}
$$

Equations (3.10), (3.11) and (3.12) provide three relationships between $\beta_{1}$ and $\beta_{2}$, so in theory it should be possible to eliminate them and get an equation for $\alpha$. In practice, it is more convenient to look for further linear relationships between $\beta_{1}$ and $\beta_{2}$.

If we insert $\delta$ from equation (3.9) into equation (2.21), the only non-linear term in $\beta_{1}$ and $\beta_{2}$ is $\beta_{1} \beta_{2}$ and this can be replaced by linear terms from equation (3.12). This leads to a linear equation

$$
\begin{aligned}
& \left\{p(2 m-2 \alpha)+1+m^{2}\right\} \beta_{1}+\left\{p(4 m-2 \alpha)-\alpha^{2}+8 m \alpha-10 m^{2}+2\right\} \beta_{2} \\
& =\left(1+m^{2}\right)\left(p^{2}+\alpha^{2}+4\right)+p\left\{-\alpha^{3}+4 m \alpha^{2}-\left(1+13 m^{2}\right) \alpha-8 m+8 m^{3}\right\} .
\end{aligned}
$$

We can obtain a slightly neater alternative if we use equation (3.10) to replace $p \beta_{1}(2 m-2 \alpha)$. This leaves

$$
\begin{aligned}
(1+ & \left.m^{2}\right) \beta_{1}+\left(2 m p+\alpha^{2}-6 m \alpha+2 m^{2}+2\right) \beta_{2} \\
= & \left(1+m^{2}\right)\left(p^{2}+\alpha^{2}+4+8 m^{2}-8 m \alpha\right) \\
& +p\left\{\alpha^{3}-10 m \alpha^{2}+\left(3+35 m^{2}\right) \alpha-12 m-28 m^{3}\right\} .
\end{aligned}
$$

There are several ways of obtaining a third linear relationship between $\beta_{1}$ and $\beta_{2}$. Subtracting equation (2.10) from equation (2.9) gives

$$
\left(1+m^{2}\right)\left\{\sum b_{1}^{2} b_{2} b_{3}-\sum b_{1} b_{2} b_{3}^{2}\right\}=2 k\left\{\gamma-\sum b_{1} b_{2}^{2}\right\}
$$

and we can use equations (2.5), (3.5), (2.13) and (2.4) to replace the summations. This brings in terms in $\gamma$ and $\delta$ but equation (3.2) and (3.9) allow them to be expressed in terms of $\beta_{1}$ and $\beta_{2}$. The resulting linear equation is fairly cumbersome but by using equations (3.10) and (3.14) it reduces to

$$
\begin{aligned}
3 p^{2} \beta_{1} & +\left\{2 p^{2}+(\alpha-4 m) p-\alpha^{2}+10 m \alpha-2-26 m^{2}\right\} \beta_{2} \\
= & p^{2} \alpha^{2}+\left(1+m^{2}\right)\left(2 p^{2}-\alpha^{2}+10 m \alpha-4-28 m^{2}\right) \\
& +p\left\{-\alpha^{3}+14 m \alpha^{2}-\left(3+67 m^{2}\right) \alpha+16 m+112 m^{3}\right\} .
\end{aligned}
$$

In general, equations (3.10), (3.14) and (3.15) are linearly independent and lead to a cubic equation for $\alpha$. 
If we eliminate $\beta_{1}$ from equations (3.10) and (3.14), we get an equation of the form

$$
L_{1} \beta_{2}=N_{1}
$$

where

$$
\begin{aligned}
L_{1}=2 m p^{2}+p\left(\alpha^{2}-6 m \alpha+1+m^{2}\right)-(\alpha-6 m)\left(1+m^{2}\right), & \\
N_{1}= & p^{3}\left(1+m^{2}\right)+p^{2}\left\{\alpha^{3}-10 m \alpha^{2}+\left(3+35 m^{2}\right) \alpha-12 m-28 m^{3}\right\} \\
& +p\left(1+m^{2}\right)\left(2-10 m^{2}-2 m \alpha\right)+4 m\left(1+m^{2}\right)^{2} .
\end{aligned}
$$

In the same way, if we eliminate $\beta_{1}$ from equations (3.10) and (3.15) we get

$$
L_{2} \beta_{2}=N_{2} \text {, }
$$

where

$$
\begin{aligned}
L_{2}= & p^{2}+p(2 \alpha-14 m)+\left(\alpha^{2}-10 m \alpha+2+26 m^{2}\right), \\
N_{2}= & p^{2}\left(2 \alpha^{2}-18 m \alpha+4+52 m^{2}\right) \\
& +p\left\{\alpha^{3}-14 m \alpha^{2}+\left(3+67 m^{2}\right) \alpha-28-124 m^{3}\right\} \\
& +\left(1+m^{2}\right)\left(\alpha^{2}-10 m \alpha+4+28 m^{2}\right) .
\end{aligned}
$$

For equations (3.16) and (3.19) to be consistent, the condition is that

$$
G(\alpha, m, p) \equiv L_{2} N_{1}-L_{1} N_{2}=0 \text {. }
$$

From the expressions for $L_{1}, L_{2}, N_{1}$ and $N_{2}$,

$$
G(\alpha, m, p)=g_{0} \alpha^{3}+g_{1} \alpha^{2}+g_{2} \alpha+g_{3},
$$

with

$$
\begin{aligned}
g_{0}= & p^{4}-6 m p^{3}+\left(3+11 m^{2}\right) p^{2}-6 m p\left(1+m^{2}\right)+\left(1+m^{2}\right)^{2} \\
= & \left(p^{2}-3 m p+1+m^{2}\right)^{2}+p^{2} \\
g_{1}= & -14 m p^{4}+\left(1+77 m^{2}\right) p^{3}-\left(38 m+134 m^{3}\right) p^{2} \\
& +72 m^{2}\left(1+m^{2}\right) p-12 m\left(1+m^{2}\right)^{2} \\
g_{2}= & \left(5+73 m^{2}\right) p^{4}-\left(42 m+362 m^{3}\right) p^{3}+\left(11+226 m^{2}+567 m^{4}\right) p^{2} \\
- & m\left(1+m^{2}\right)\left(28+292 m^{2}\right) p+\left(4+48 m^{2}\right)\left(1+m^{2}\right)^{2} \\
g_{3}= & p^{5}\left(1+m^{2}\right)-m p^{4}\left(34+146 m^{2}\right)+m^{2} p^{3}\left(188+604 m^{2}\right) \\
& -m p^{2}\left(52+496 m^{2}+828 m^{4}\right)+m^{2} p\left(1+m^{2}\right)\left(112+400 m^{2}\right) \\
& -16 m\left(1+4 m^{2}\right)\left(1+m^{2}\right)^{2} .
\end{aligned}
$$

From equation (3.24), $g_{0}$ is positive for $p>0$ and indeed it is straightforward to show that $g_{0}$ has a minimum value $16 / 25$. Hence $G(\alpha, m, p)=0$ gives a cubic equation for $\alpha$ for any pair $(m, p)$. Also, for $m \leqslant 0$ and $p>0, g_{0}, g_{1}, g_{2}$ and $g_{3}$ 
are all positive and in this case $G=0$ cannot have a positive root. This rules out a $C 4$ solution since we need $b_{i}>0$ for a periodic solution. (From equation (1.1), we have $0<y_{1} \leqslant 2 k$ for all values of $y_{0}$ and indeed $0<y_{n} \leqslant 2 k$ for $n \geqslant 1$. This means that in looking for equilibrium solutions or periodic solutions, we can take $0 \leqslant y_{0} \leqslant 2 k$ and regard the iteration as a mapping of an interval into itself.)

\section{Stability criterion and solution for cyclic elements}

For given values of $m$ and $k$, we have $p=2 k /\left(1+m^{2}\right)$ and $g_{0}, g_{1}, g_{2}, g_{3}$ can be calculated. The only problem is that we may be losing some accuracy by taking the difference between large terms. For example, when $m=3$

$$
g_{2}=662 p^{4}-9900 p^{3}+47972 p^{2}-79680 p+43600
$$

and we want to use values of $p$ between 0 and 6 . For some of the calculations a change of origin was made to alleviate this problem. When $g_{0}$ to $g_{3}$ have been found, the next step is to solve the cubic equation for $\alpha$ and this was done by using the iteration procedure developed in Section 7 of Paper I. For each real positive $\alpha$, equation (3.16) or equation (3.19) gives $\beta_{2}$ (provided $L_{1}$ and $L_{2}$ are not too close to zero) and equation (3.14) then gives $\beta_{1}$. Equations (3.2) and (3.9) give $\gamma$ and $\delta$, while $\beta$ is simply $\beta_{1}+\beta_{2}$. Thus we can form $h(y)$, as given by equation (2.3), and solve $h(y)=0$ to obtain $b_{1}, b_{2}, b_{3}$ and $b_{4}$. Some comments on this are given below. It was also convenient to evaluate the stability criterion, $S$, for each $C 4$ solution after $\alpha, \beta, \gamma$ and $\delta$ had been determined. The stability criterion is

$$
S=F^{\prime}\left(b_{1}\right) F^{\prime}\left(b_{2}\right) F^{\prime}\left(b_{3}\right) F^{\prime}\left(b_{4}\right),
$$

and $S$ must be between -1 and +1 for local stability. From equations (1.1) and (2.2)

$$
F^{\prime}\left(b_{1}\right)=\frac{-4 k\left(b_{1}-m\right)}{\left\{1+\left(b_{1}-m\right)^{2}\right\}^{2}}=\frac{4 k\left(m-b_{1}\right)}{\left(2 k / b_{2}\right)^{2}}=\frac{b_{2}^{2}\left(m-b_{1}\right)}{k} .
$$

It follows that

$$
\begin{aligned}
S & =\left(b_{1} b_{2} b_{3} b_{4}\right)^{2}\left(m-b_{1}\right)\left(m-b_{2}\right)\left(m-b_{3}\right)\left(m-b_{4}\right) / k^{4} \\
& =\left(\delta^{2} / k^{4}\right) h(m) .
\end{aligned}
$$

Thus $S$ can be evaluated without knowing $b_{1}, b_{2}, b_{3}, b_{4}$ explicitly and indeed $S$ can be found and used even for cases where the solution for the $b_{i}$ is complex. (This occurs when the transition from $S>1$ to $S<1$ marks the transition from complex to real solutions.) 
In solving for the cyclic elements, we note from equations (2.19) and (2.20) that $2 m-b_{1}-b_{3}$ and $2 m-b_{2}-b_{4}$ must have opposite signs. (From equation (2.21) their product, $4 m^{2}-2 m \alpha+\beta_{1}$, is non-zero and negative.) If we take $b_{1}+b_{3}>$ $2 m$, then we must have

$$
b_{1}+b_{3}>2 m>b_{2}+b_{4} \text {. }
$$

Now $b_{1}+b_{3}$ and $b_{2}+b_{4}$ are the roots of the quadratic

$$
0=\left(X-b_{1}-b_{3}\right)\left(X-b_{2}-b_{4}\right)=X^{2}-\alpha X+\beta_{1},
$$

and for real, distinct roots we must have $\alpha^{2}>4 \beta_{1}$. If this condition is satisfied we can take $b_{1}+b_{3}$ as the larger root of equation (4.5), with $b_{2}+b_{4}$ as the smaller root. This is simply a matter of how we label the elements and in the same way we can assume that $b_{1}$ is the larger of the pair $b_{1}$ and $b_{3}$. From equation (2.20), $b_{1}>b_{3}$ then implies that $b_{4}>b_{2}$. If we write

$$
X_{1}=b_{1}+b_{3}, \quad X_{2}=b_{2}+b_{4}, \quad Y_{1}=b_{1} b_{3}, \quad Y_{2}=b_{2} b_{4},
$$

then a knowledge of $X_{1}$ and $Y_{1}$ is enough to give $b_{1}$ and $b_{3}$, and a knowledge of $X_{2}$ and $Y_{2}$ is enough to give $b_{2}$ and $b_{4}$. We can say that $Y_{1}$ and $Y_{2}$ are the roots of the quadratic

$$
0=\left(Y-b_{1} b_{3}\right)\left(Y-b_{2} b_{4}\right)=Y^{2}-\beta_{2} Y+\delta
$$

but unfortunately we do not know whether $b_{1} b_{3}$ or $b_{2} b_{4}$ is the larger root. (Again, $\beta_{2}^{2} \geqslant 4 \delta$ is a necessary condition for real roots.) However we can get around this difficulty by using equations (2.19) and (2.20) to obtain

$$
\begin{aligned}
& 2 k b_{1} b_{3}\left(b_{4}-b_{2}\right)^{2}\left(2 m-b_{2}-b_{4}\right) \\
& \quad=2 k b_{2} b_{4}\left(b_{1}-b_{3}\right)^{2}\left(b_{1}+b_{3}-2 m\right) .
\end{aligned}
$$

This gives

$$
\left.Y_{1}\left\{\left(2 m-X_{2}\right) X_{2}^{2}\right\}-Y_{2}\left\{X_{1}-2 m\right) X_{1}^{2}\right\}=4 \delta(4 m-\alpha) .
$$

Also, from equation (2.14),

$$
\begin{aligned}
\alpha \beta_{2}-\gamma & =b_{1} b_{3}\left(b_{1}+b_{3}\right)+b_{2} b_{4}\left(b_{2}+b_{4}\right) \\
& =Y_{1} X_{1}+Y_{2} X_{2} .
\end{aligned}
$$

From these two equations

$$
\begin{aligned}
& Y_{1}\left\{X_{1}^{3}\left(X_{1}-2 m\right)+X_{2}^{3}\left(2 m-X_{2}\right)\right\} \\
& \quad=4 \delta(4 m-\alpha) X_{2}+\left(\alpha \beta_{2}-\gamma\right) X_{1}^{2}\left(X_{1}-2 m\right),
\end{aligned}
$$

with a similar equation for $Y_{2}$. The equation $Y_{1}+Y_{2}=\beta_{2}$ might have been used as an alternative to equation (4.9) but instead it was incorporated in a check, which was to evaluate $Y_{1}^{2}-\beta_{2} Y_{1}+\delta$ and $Y_{2}^{2}-\beta_{2} Y_{2}+\delta$ and ensure that they were close to zero. (From equation (4.7), these expressions should be exactly zero.) 
To find $b_{1}$ and $b_{3}$ we have to solve the quadratic

$$
0=\left(Z-b_{1}\right)\left(Z-b_{3}\right)=Z^{2}-X_{1} Z+Y_{1}
$$

and for real distinct roots we must have

$$
\left(b_{1}-b_{3}\right)^{2}=X_{1}^{2}-4 Y_{1}>0 .
$$

If this condition is satisfied, we can take

$$
b_{1}-b_{3}=\sqrt{ }\left(X_{1}^{2}-4 Y_{1}\right) \text {, }
$$

and in the same way we can take

$$
b_{4}-b_{2}=\sqrt{ }\left(X_{2}^{2}-4 Y_{2}\right),
$$

provided $X_{2}^{2}>4 Y_{2}$. Thus the solution for $b_{1}, b_{2}, b_{3}$, and $b_{4}$ can be obtained without solving $h(y)=0$ as a quartic equation. The knowledge of $\beta_{1}$ and $\beta_{2}$ allows us to compute the $b_{i}$ via linear and quadratic equations.

The procedure given above was adequate in most cases although some modification was made for a few values of $m$ and $k$ where special difficulties arose. These difficulties are probably easier to explain in relation to the general run of the numerical results, which are discussed in the next section.

\section{Numerical results}

The computational work was carried out on a Univac 1100 computer, using double precision for all variables. A number of numerical checks were included and at the end the quantities

$$
\begin{aligned}
& e_{t}=b_{1+1}-F\left(b_{1}\right) \quad(i=1,2,3), \\
& e_{4}=b_{1}-F\left(b_{4}\right)
\end{aligned}
$$

were calculated. The $e$ 's represent deviations from an exact fit to equation (1.1) and provide an obvious check on the accuracy of the solutions for $b_{1}, b_{2}, b_{3}, b_{4}$. The normal practice was to evaluate $\beta_{2}$ from equation (3.16) and from equation (3.19), then to use the mean of the two estimates in the later calculations unless some discrepancy was apparent. It became clear that $L_{1}$ and $L_{2}$ could be zero for some values of $m$ and $k$, so a lower limit of 0.8 was imposed on the modulus of these quantities. When $\left|L_{1}\right|$ and $\left|L_{2}\right|$ were both less than $0.8, \beta_{2}$ was obtained from a quadratic equation

$$
(6 m-\alpha-p) \beta_{2}^{2}-v_{1} \beta_{2}+v_{2}=0,
$$


where

$$
\begin{gathered}
v_{1}=\alpha^{3}-(p+12 m) \alpha^{2}+\left(2+46 m^{2}+6 m p\right) \alpha \\
-48 m^{3}-20 m^{2} p-4 p \\
v_{2}=p\left\{\alpha^{4}-16 m \alpha^{3}+\left(2+94 m^{2}\right) \alpha^{2}\right. \\
\left.-\left(20 m+244 m^{3}\right) \alpha-4+24 m^{2}+220 m^{4}\right\} \\
+\left(1+m^{2}\right)\left\{\alpha^{3}-12 m \alpha^{2}+\left(4+48 m^{2}-p^{2}\right) \alpha-8 m-56 m^{3}+12 m p^{2}\right\}
\end{gathered}
$$

This quadratic comes from equation (3.12) for $\beta_{1} \beta_{2}$. If we multiply equation (3.12) by $p$, we can replace $p \beta_{1}$ on the left-hand side from equation (3.10) and on the right-hand side we can replace $3 p^{2} \beta_{1}+2 p^{2} \beta_{2}$ from equation (3.15). This eliminates $\beta_{1}$ and leaves the quadratic equation for $\beta_{2}$.

Equation (5.2) gives two values for $\beta_{2}$ and we can use equation (3.14) to determine the corresponding values for $\beta_{1}$. Each pair was then substituted in equation (3.11) and the pair which gave better agreement was taken as the appropriate pair in the subsequent calculations. (Occasionally other information was taken into account in deciding which pair to use.) Once $\beta_{1}$ and $\beta_{2}$ had been found the solution for $\gamma, \delta, S, b_{i}, e_{i}$ followed as before.

Although no real $C 4$ solutions were expected for $m<1$, some runs were made with $m$ between 0 and 1 and with $k$ between 0.25 and 3.0. In each case there was a single real value for $\alpha$ but the solution for the elements $b_{i}$ was complex, with $S>1$. It was clear that, for a given value of $m, S$ had a minimum around $k=2.0$. The minimum value decreased as $m$ increased, with a minimum of 1.8 , 1.4 and 1.1 recorded for $m=0.9,0.95$ and 0.99 , respectively. For $m=1.0$ and $k=2.0$, a solution with $S=1$ was recorded and for $m>1$ real solutions appeared for some values of $k$.

As mentioned in Section 1, there is a $C 2$ solution which has $S_{2}=-1$ for $m=1$ and $k=2$. Its elements are $b_{1}=2+\sqrt{ } 2$ and $b_{2}=2-\sqrt{ } 2$ and we can think of it as the limiting case of a $C 4$ solution which has $b_{1}=b_{3}, b_{2}=b_{4}$ and $S=(-1)^{2}=1$. The computed $C 4$ solution for $m=1$ and $k=2$ gave values of $\alpha, \beta, \gamma, \delta$, $\left(b_{1}-b_{3}\right)^{2}$ and $\left(b_{2}-b_{4}\right)^{2}$ which agreed with this interpretation (to 12 decimal places), although the solution for the $b_{1}$ was recorded as complex. Presumably $\left(b_{1}-b_{3}\right)^{2}$ and $\left(b_{2}-b_{4}\right)^{2}$ came out as very small negative numbers and the programme stopped (as instructed) when that happened.

This difficulty appeared in a slightly different form for $m>1$ and $k$ close to $k_{5}$ or $k_{6}$. For example, for $k$ slightly greater than $k_{5}$ the computations gave a real

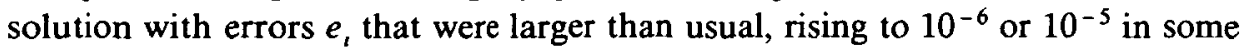
instances. This appeared to be because $b_{1}-b_{3}$ and $b_{2}-b_{4}$ were close to zero and the calculated values of $\left(b_{1}-b_{3}\right)^{2}$ and $\left(b_{2}-b_{4}\right)^{2}$ had errors of about the same 
size as the calculated values, say about $10^{-12}$. When the square root was taken to obtain $b_{1}-b_{3}$ and $b_{4}-b_{2}$, the square root had an error of order $10^{-6}$.

For $m>1$, real $C 4$ solutions were obtained for $k_{5}<k<k_{6}$. The cubic equation for $\alpha$ always has at least one real root, and for $m<m_{2}=2.305976$ there was only one real root. We can call this the $\alpha_{0}$ root, and refer to the corresponding solutions for $b_{i}$ as the $\alpha_{0}$ family or main family of solutions. For this family, $S$ was greater than 1 for $k<k_{5}$ and for $k>k_{6}$ and the solution for the $b_{2}$ was then complex. For $k_{5}<k<k_{6}, S$ decreased rapidly from +1 to a minimum and then increased again more slowly, with $S \rightarrow 1$ as $k \rightarrow k_{6}$. For $1<m<m_{1}=1.202517$ the minimum was greater than -1 so there was a single interval of stability $\left(k_{5}, k_{6}\right)$. For $m>m_{1}$ the minimum was less than -1 and there were two intervals of stability $\left(k_{5}, k_{5}^{*}\right)$ and $\left(k_{6}^{*}, k_{6}\right)$, where $k_{5}^{*}$ and $k_{6}^{*}$ are the values cf $k$ for which $S=-1$. Some values for $k^{*}, k_{5}, k_{5}^{*}, k_{6}^{*}, k_{6}$ are given in Table 1 and it will be seen that the length of the $\left(k_{5}, k_{5}^{*}\right)$ interval is tending to decrease as $m$ increases, but the $\left(k_{6}^{*}, k_{6}\right)$ interval is getting larger.

TABLE 1. Critical values of $k$ (for a given $m$ )

Main family of $C 4$ solutions Additional $C 4$ solutions

\begin{tabular}{c|c||cccc||cccc}
$m$ & $k^{*}$ & $k_{5}$ & $k_{5}^{*}$ & \multicolumn{1}{c}{$k_{6}^{*}$} & \multicolumn{1}{c}{$k_{6}$} & $k_{7}$ & $k_{7}^{*}$ & $k_{8}^{*}$ & $k_{8}$ \\
1.00 & 0.8284 & 2.0000 & - & - & 2.0000 & - & - & - & - \\
1.25 & 0.8989 & 1.2812 & 1.6125 & 2.7762 & 5.1250 & - & - & - & - \\
1.50 & 0.9840 & 1.2414 & 1.3593 & 5.1146 & 8.5086 & - & - & - & - \\
2.00 & 1.1803 & 1.3397 & 1.3918 & 11.2151 & 18.6603 & - & - & - & - \\
2.50 & 1.3962 & 1.5132 & 1.5468 & 20.5265 & 34.7368 & 1.879595 & 1.888580 & 3.12443 & 3.44141 \\
3.00 & 1.6228 & 1.7157 & 1.7409 & 33.9905 & 58.2842 & 1.917941 & 1.919330 & 5.27415 & 6.10187 \\
3.50 & 1.8557 & 1.9331 & 1.9533 & 52.4775 & 90.8169 & 2.078314 & 2.078917 & 8.00027 & 9.57957 \\
4.00 & 2.0928 & 2.1593 & 2.1762 & 76.8405 & 133.8407 & 2.274070 & 2.274430 & 11.52553 & 14.11337
\end{tabular}

$$
m_{1}=1.202517, \quad m_{2}=2.305976, \quad m_{3}=2.334496 .
$$

For $m>m_{2}$, three real solutions occurred over an interval $k_{7}<k<k_{8}$, where $k_{7}$ and $k_{8}$ were determined numerically for various values of $m$. One of these solutions was the $\alpha_{0}$ solution, which was unstable in $\left(k_{7}, k_{8}\right)$, and two additional families of solutions were recognisable. For one of these additional families, $S$ was greater than 1 in $\left(k_{7}, k_{8}\right)$ and we can refer to these solutions as the unstable additional family. For this family, $S \rightarrow 1$ as $k \rightarrow k_{7}$ and as $k \rightarrow k_{8}$, with $S$ rising to a maximum at some intermediate value of $k$. For the other additional family, $S=1$ at $k=k_{7}$, with $S$ decreasing to a minimum at an intermediate value of $k$ and approaching 1 again as $k \rightarrow k_{8}$. We can refer to this as the "stable" family of additional solutions since it has $|S|<1$ for some values of $k$. For $m>m_{3}=$ 2.334496 , the minimum value of $S$ was less than -1 and hence there were two intervals of stability, say $\left(k_{7}, k_{7}^{*}\right)$ and $\left(k_{8}^{*}, k_{8}\right)$, where $k_{7}^{*}$ and $k_{8}^{*}$ are the values of $k$ at which $S=-1$ for this family of solutions. For $m_{2}<m<m_{3}$ the minimum $S$ 
was greater than -1 and the interval of stability was then $\left(k_{7}, k_{8}\right)$. Some numerical values are given in Table 1 and for comparison the corresponding critical values for $C 3$ solutions are listed in Table 2. (The notation is that $C 3$ solutions occur for $K_{1} \leqslant k \leqslant K_{2}$ and one of the solutions is stable for $K_{1}<k<$ $K_{1}^{*}$ and for $K_{2}^{*}<k<K_{2}$.)

At $k=k_{7}$ and $k=k_{8}$, the two additional solutions coalesce to give a single solution, with $S=1$.

TABLE 2. Critical values of $k$ (for a given $m$ )

C3 solutions

\begin{tabular}{ccccc}
$m$ & $K_{1}$ & $K_{1}^{*}$ & $K_{2}^{*}$ & \multicolumn{1}{c}{$K_{2}$} \\
$\sqrt{3}$ & 2.3094 & - & - & 2.3094 \\
2.0 & 1.6667 & 1.7503 & 2.9784 & 5.0000 \\
2.5 & 1.6850 & 1.7014 & 5.6889 & 10.3984 \\
3.0 & 1.8350 & 1.8432 & 9.2799 & 18.1650 \\
3.5 & 2.0256 & 2.0308 & 14.1180 & 28.8911 \\
4.0 & 2.2352 & 2.2391 & 20.4424 & 43.0981
\end{tabular}

In determining the critical values for $m$ and $k$, it was often useful to improve the solutions for the elements $b$ by a simple but effective device. If we have a value $b_{1}$ which is an approximation to the exact value $b_{1}^{*}$ for an element for the $C 4$ solution then we can iterate equation (1.1) four times to obtain, say $b_{5}$. Now, to the first order in $b_{1}-b_{1}^{*}$, the iteration should give

$$
b_{5}-b_{1}^{*}=S\left(b_{1}-b_{1}^{*}\right) \text {, }
$$

and it follows that

$$
(1-S) b_{1}^{*}=b_{5}-S b_{1} \text {. }
$$

In general, this gives an improved estimate of $b_{1}^{*}$, and we can use this improved estimate to re-calculate $b_{2}, b_{3}, b_{4}, \alpha, \beta, \gamma, \delta$ and $S$. The procedure can then be repeated and the solution errors $e_{\text {, }}$ calculated to monitor the process. One interesting point is that the $C 4$ solution need not be stable; a large value of $|S|$ becomes an advantage rather than a nuisance. Clearly, equation (5.6) does not help when $S=1$ but in practice the equation was used with $1-S$ of order $10^{-6}$, without any difficulty arising. (Occasionally there were indications that the iterations were settling into periodic solutions of small amplitude for $S$.)

\section{Special cases}

In the derivation of equation (3.9) from equation (3.8), it was assumed that $\alpha-4 p$ was non-zero and it was noted also that a proviso $\alpha \neq 8 m$ was needed. From the numerical work, solutions with $\alpha=4 p$ and solutions with $\alpha=8 m$ occur, although there was no indication that anything unusual happened in either 
case. To check this, it was assumed that $\alpha=4 p$ and the various equations were developed on this assumption. For $p \neq 2 m$, the basic equation for $\alpha$ was replaced by an equation linking $p$ and $m$, which proved to be the same as $G(4 p, m, p)=0$ [equation (3.22)].

In the same way, assuming $\alpha=8 m \neq 4 p$ led to an equation between $p$ and $m$ which was simply $G(8 m, m, p)=0$. The other equations used in the solution were also unaffected.

For the special case $\alpha=4 p=8 m$, equation (2.2) is replaced by

$$
\left(2 m-b_{2}\right)\left(1+m^{2}\right)=b_{1}^{2} b_{2}-2 m b_{1} b_{2}
$$

and several of the equations are simpler. For example,

$$
\begin{aligned}
& \sum b_{1}^{2} b_{2}=\sum b_{1} b_{2}^{2}=\gamma=2 m \beta_{1}, \\
& \beta_{1} \beta_{2}=\left(6 m^{2}-2\right) \beta_{1}+32 m^{2}\left(1+m^{2}\right), \\
& 2 \delta=\left(1+3 m^{2}\right) \beta_{1}+\left(1+m^{2}\right) \beta_{2}-24 m^{2}\left(1+m^{2}\right), \\
& \beta_{1}^{2}=4 \delta+\left(2+14 m^{2}\right) \beta_{1}-\left(2+2 m^{2}\right) \beta_{2}-16 m^{2}\left(1+m^{2}\right) \\
& \quad=\left(4+20 m^{2}\right) \beta_{1}-64 m^{2}\left(1+m^{2}\right) .
\end{aligned}
$$

Equation (6.5) gives either $\beta_{1}=16 \mathrm{~m}^{2}$ or $\beta_{1}=4+4 \mathrm{~m}^{2}$.

For $\beta_{1}=16 \mathrm{~m}^{2}$, we get $\beta_{2}=8 \mathrm{~m}^{2}, \gamma=32 \mathrm{~m}^{3}$ and $\delta=16 \mathrm{~m}^{4}$ and the corresponding solution is an equilibrium solution with $b_{1}=b_{2}=b_{3}=b_{4}=2 \mathrm{~m}$. In similar fashion, $\beta_{1}=4+4 m^{2}$ leads to

$$
\begin{aligned}
& \beta_{2}=14 m^{2}-2, \quad \beta=2+18 m^{2}, \\
& \gamma=8 m\left(1+m^{2}\right), \quad \delta=\left(1+m^{2}\right)^{2}
\end{aligned}
$$

and the equation $h(X)=0$ for the cyclic elements becomes

$$
\begin{aligned}
0 & =X^{4}-8 m X^{3}+\left(2+18 m^{2}\right) X^{2}-8 m\left(1+m^{2}\right) X+\left(1+m^{2}\right)^{2} \\
& =\left(X^{2}-4 m X+1+m^{2}\right)^{2} .
\end{aligned}
$$

For $m^{2}>1 / 3$, the solution is real but degenerate, with

$$
b_{1}=b_{3}=2 m+\sqrt{ }\left(3 m^{2}-1\right), \quad b_{2}=b_{4}=2 m-\sqrt{ }\left(3 m^{2}-1\right) .
$$

Thus the condition $\alpha=4 p=8 m$ does not lead to a non-degenerate solution of period 4.

For the numerical work the special cases $\alpha=4 p$ and $\alpha=8 m$ were of no consequence but, as mentioned in Section 5 , the computational technique had to be altered when $L_{1}$ and $L_{2}$ were close to zero. This suggested that it would be worth examining points where $L_{1}=0$ and $L_{2}=0$ and finding what happens to the $C 4$ solutions at these points. From equations (3.17) and (3.20), we are concerned with points at which

$$
\begin{aligned}
& p \alpha^{2}-\left(6 m p+1+m^{2}\right) \alpha+2 m p^{2}+(p+6 m)\left(1+m^{2}\right)=0, \\
& \alpha^{2}+(2 p-10 m) \alpha+p^{2}-14 m p+26 m^{2}+2=0 .
\end{aligned}
$$


Eliminating $\alpha$ gives an equation $Q(m, p)=0$, where

$$
\begin{aligned}
Q(m, p)= & p^{6}-12 m p^{5}+\left(8+64 m^{2}\right) p^{4} \\
& -\left(46 m+110 m^{3}\right) p^{3}+\left(8+80 m^{2}+72 m^{4}\right) p^{2} \\
& -20 m\left(1+m^{2}\right)^{2} p+2\left(1+m^{2}\right)^{3} .
\end{aligned}
$$

The equation for $\alpha$ can be written as

$$
\left(2 p^{2}-4 m p+1+m^{2}\right) \alpha=-p^{3}+16 m p^{2}-\left(1+25 m^{2}\right) p+6 m\left(1+m^{2}\right) .
$$

For a finite $C 4$ solution, $\beta_{2}$ must be finite and this implies that $L_{1}$ cannot be zero without having $N_{1}$ zero also (from equation (3.16)). In the same way, $N_{2}$ should be zero when $L_{2}$ is zero. It can be verified that the condition $Q(m, p)=0$ also arises from taking $L_{1}=0, L_{2}=0, N_{2}=0$ simultaneously and from the simultaneous equations $L_{1}=0, L_{2}=0, N_{1}=0, N_{2}=0$. The numerical work agrees with this.

\begin{tabular}{c|rrrr}
\multicolumn{5}{c}{ TABLE 3. Values of $m$ and $k$ for which $L_{1}=0, L_{2}=0$} \\
$m$ & \multicolumn{3}{c}{ Values of $k$} \\
1.25 & 1.175541, & 1.444044 & & \\
1.50 & 1.272026, & 2.712829 & & \\
2.00 & 2.041693, & 6.130276 & & \\
2.50 & 3.314790, & 11.499324 & & \\
3.00 & 5.166206, & $19.357997 ;$ & 5.719589, & 6.101476 \\
3.50 & 7.699261, & $30.215996 ;$ & 8.329993, & 9.425541 \\
4.00 & 11.022819, & $44.577753 ;$ & 11.829435, & 13.886449
\end{tabular}

Since $G(\alpha, m, p)=L_{2} N_{1}-L_{1} N_{2}$, it follows that $G$ and its first order partial derivatives are zero at points where $L_{1}, L_{2}, N_{1}$ and $N_{2}$ are all zero. However, although this is a sufficient condition for a double root it is not a necessary condition. Also, the double root for $\alpha$ need not lead to a real solution for $b_{1}$ to $b_{4}$. For the double root, $\alpha$ is given by equation (6.10) and, for given values of $m$ and $p$, there will be another real value of $\alpha$ for which $L_{1}, L_{2}, N_{1}$ and $N_{2}$ are non-zero even although $Q(m, p)=0$.

Table 3 lists some values of $m$ and $k$ for which $Q(m, p)=0$, and it will be seen that there are two values of $k$ for $m=1.25,1.50,2.00$ and 2.50 , with two additional values of $k$ for larger values of $m$. The numerical calculations give two critical values for $m$, namely

$$
m_{11}=1.233603, \quad m_{12}=2.880208,
$$

and for a given value of $m$ the equation $Q(m, p)=0$ has no real roots for $1<m<m_{11}$, two real roots for $m_{11}<m<m_{12}$ and four real roots for $m>m_{12}$. Some of these roots occur in $(m, k)$ regions where no real $C 4$ solutions are 
expected (for example, $m=1.25, k=1.175541$ ), others where a single real $C 4$ solution is expected (for example, $m=1.50, k=2.712829$ ) and others where three real $C 4$ solutions are expected (for example, $m=3.00, k=5.719589$ ). It turns out that the occurrence of these special points does not change the general picture, although they cause some anomalous results in the numerical work.

For example, for $m=1.25$ and $k$ going from 1.17551 to 1.17558 , in steps of $10^{-5}$, the equation for $\alpha$ has one real root near 6.78, well away from the value of $\alpha$ given by equation (6.10). For this value of $\alpha, L_{1}$ and $L_{2}$ are not close to zero and the solution goes through in the usual way. (It comes out as a complex solution for the $b_{i}$ ). The equation for $\alpha$ also has two complex roots, $g \pm h i$, with the imaginary part $h$ of order $10^{-5}$ or $10^{-6}$. If we take $h$ as positive, $h$ should decrease to zero as $k$ goes through its critical value and then increase again, thus providing a double real root when $h=0$. The double root should give the value of $\alpha$ required by equation (6.10). The numerical work agreed with this reasonably well, although for $k=1.17554$ the equation for $\alpha$ gave three real roots. Two of these differed by $2 \times 10^{-6}$, which is about as close to a double root as we might expect. When these values of $\alpha$ were used in equation (5.2) they gave complex values for $\beta_{2}$ and the solution for the $b_{1}$ stopped at this point.

Similar results were obtained in other cases where a single real solution for $\alpha$ occurred at almost all neighbouring points. Where there were three real solutions for $\alpha$ at almost all neighbouring points, the double root corresponded to a "cross-over" of two of these solutions. (In Paper I, behaviour of this type was mentioned for the $C 3$ solutions at $k=K_{0}$.) For example, if we take $m=3.0$ and $k$ close to 5.166206, the main family of solutions has $S \doteqdot-116$, the additional stable family has $S \doteqdot-1.25$ and the additional unstable has $S \doteqdot+10.5$. If we use $\alpha_{0}, \alpha_{1}$ and $\alpha_{2}$ for the corresponding values of $\alpha$, then $\alpha_{0}>\alpha_{2}>\alpha_{1}$ for $k=$ 5.166201 and $\alpha_{0}>\alpha_{1}>\alpha_{2}$ for $k=5.166211$, with $\alpha_{1}=\alpha_{2}$ at the point where $L_{1}$ and $L_{2}$ are zero. The values of $\beta_{1}, \beta_{2}, \gamma, \delta$ and $S$ are appreciably different for the $\alpha_{1}$ and $\alpha_{2}$ families in this neighbourhood so there is no difficulty in distinguishing them. In the same way, there is a cross-over point for $m=3.0$ and $k=5.719589$, where the pattern $\alpha_{0}>\alpha_{1}>\alpha_{2}$ changes to $\alpha_{1}>\alpha_{0}>\alpha_{2}$ as $k$ increases, with $\alpha_{0}=\alpha_{1}$ at the cross-over point. There is another cross-over point at $m=3.0$ and $k=6.101476$, where $\alpha_{1}>\alpha_{0}>\alpha_{2}$ changes back to $\alpha_{0}>\alpha_{1}>\alpha_{2}$ as $k$ increases. Again, $\alpha_{0}=\alpha_{1}$ at the cross-over point, with the corresponding $C 4$ solutions clearly distinguishable.

In contrast, we can compare what happens at $m=3.0, k=k_{8}=6.10187$. At this point, $\alpha_{1}=\alpha_{2}$ and the equation for $\alpha$ again has a double root but as $k$ increases through $k_{8}$ we have a transition from three real solutions for $\alpha$ to a single real solution. The $\alpha_{0}$ solution continues but $\alpha_{1}$ and $\alpha_{2}$ become complex for $k>k_{8}$. At the transition point the two additional solutions merge into a single 
solution, with $S=1$ and with $L_{1}$ and $L_{2}$ non-zero. This is a reminder that $Q(m, p)=0$ provides a sufficient condition for a double root for $\alpha$ but is not a necessary condition.

In examining the solutions near points where $L_{1}$ and $L_{2}$ are zero, the stability criterion $S$ was an important item of information, since it helped to discriminate between different families of solutions and was usually enough in itself to classify a solution unequivocally. In addition, it allowed solutions to he improved (as described in Section 5) once a first approximation had been obtained.

\section{Discussion of results}

The main results of the paper are the critical values of $k$ in Table 1. For a given value of $m$, the $C 4$ solutions first appear at $k=k_{5}$, as the $C 2$ solutions become unstable, and the $C 4$ solutions soon become unstable as $k$ increases. In the table, the interval of stability $\left(k_{5}, k_{5}^{*}\right)$ decreases as $m$ increases and the ratio $\left(k_{5}-k^{*}\right) /\left(k_{5}^{*}-k_{5}\right)$ increases from 1.15 at $m=1.25$ to 3.93 at $m=4.0$. On the other hand, the interval $\left(k_{6}^{*}, k_{6}\right)$ becomes larger as $m$ increases and there are no $C 4$ solutions for $k>k_{6}$. For the additional solutions, there is a similar pattern. The interval of stability $\left(k_{7}, k_{7}^{*}\right)$ is becoming smaller as $m$ increases and the interval $\left(k_{8}^{*}, k_{8}\right)$ becomes larger. For the values of $m$ which give the additional intervals of stability, there are intervals of stability $\left(K_{1}, K_{1}^{*}\right)$ and $\left(K_{2}^{*}, K_{2}\right)$ for the $C 3$ solutions, with $\left(K_{1}, K_{1}^{*}\right)$ between $\left(k_{5}, k_{5}^{*}\right)$ and $\left(k_{7}, k_{7}^{*}\right)$ and with $\left(K_{2}^{*}, K_{2}\right)$ between $\left(k_{8}^{*}, k_{8}\right)$ and $\left(k_{6}^{*}, k_{6}\right)$. Behaviour of this kind was foreshadowed from the discussion of superstable $C 4$ solutions in Paper $I$, and the new results add corroborative detail.

One advantage of examining a problem with two parameters is that it may be possible to discuss a variety of one-parameter problems by linking the parameters in different ways. For example if we take $2 k=m$ in equation (1.1) and impose the condition $m>0$ (to ensure that we are dealing with positive values of $k$ ), the equation for the equilibrium values is

$$
0=Y\left\{1+(Y-m)^{2}\right\}-m=(Y-m)\left(Y^{2}-m Y+1\right) .
$$

For $0<m<2$, there is only one real equilibrium value, $Y=m$, but for $m>2$ there are three equilibrium solutions, say $Y_{1}, Y_{2}, Y_{3}$, with $0<Y_{1}<Y_{2}<Y_{3}$. We can write $Y_{3}=m$,

$$
Y_{1}=(1 / 2)\left\{m-\sqrt{ }\left(m^{2}-4\right)\right\}, \quad Y_{2}=(1 / 2)\left\{m+\sqrt{ }\left(m^{2}-4\right)\right\},
$$

and note that $Y_{1}+Y_{2}=m, Y_{1} Y_{2}=1$. From the discussion in Paper $I$, it is easy to see that $Y_{1}$ is a stable equilibrium point, $Y_{2}$ is unstable and $Y_{3}$ is stable. (For $Y=m$, the stability criterion is zero.). Also, it was shown in Paper $I$ that 
$2 k^{*}-m>0$, so in this case we have $0<k<k^{*}$ for each value of $m$. This means that there cannot be any $C 2$ solutions when $m=2 k$ and presumably no solutions with a longer period. Thus, by taking $m=2 k$, we get a one-parameter problem where the equilibrium solutions are the only periodic solutions.

If we take $k=m\left(1+m^{2}\right)$, with $m>0$, the equation for the equilibrium values is

$$
0=(Y-2 m)\left(Y^{2}+1+m^{2}\right),
$$

so the only real equilibrium solution is $Y=2 m$. The correspnding stability criterion is $S_{1}=-4 m^{2} /\left(1+m^{2}\right)$, which decreases from 0 to -4 as $m$ increases from 0 to $\infty$, with $S_{1}=-1$ for $m^{2}=1 / 3$. Thus the equilibrium solution is stable for $0<m<1 / \sqrt{ } 3$ and unstable for $m>1 / \sqrt{ }$. From the discussion of $C 2$ solutions in Paper I, there is a $C 2$ solution for $m>1 / \sqrt{ } 3$, with elements $\left(b_{1}, b_{2}\right)$ and stability criterion $S_{2}$, where

$$
b_{1,2}=2 m \pm_{\sqrt{ }}\left(3 m^{2}-1\right), \quad S_{2}=-2+\frac{1}{m^{2}} .
$$

It follows that the $C 2$ solutions are stable for $1 / \sqrt{ } 3<m<1$ and unstable for $m>1$. For $m>1$, there will be at least one $C 4$ solution if $k_{5}<k<k_{6}$ and this condition holds, since $k=m\left(1+m^{2}\right)=(1 / 2)\left(k_{5}+k_{6}\right)$. An inspection of the critical value of $k$ in Table 1 indicates that there is only one $C 4$ solution for each $m$, and that this solution is stable for $m$ slightly greater than 1 , but becomes unstable for some value of $m$ between 1.25 and 1.50 .

It looks as if the behaviour of the solutions in this case is more in line with the orthodox picture, where stable equilibrium solutions give way to stable solutions of period two as the parameter increases and these in turn give way to stable solutions of period four and so on, with stable solutions of period three appearing last of all. However it can be proved that no $C 3$ solutions are possible in this instance, so the process must come to a stop at some intermediate stage. Perhaps the simplest way of showing that no $C 3$ solutions can occur is to use the result (from Paper I) that, for a given value of $m, C 3$ solutions exist for $K_{1} \leqslant k \leqslant K_{2}$, where $m>\sqrt{ } 3$ and

$$
K_{1,2}=\frac{\left(m^{2}+1\right)}{3}\left[m \mp_{\sqrt{ }}\left(m^{2}-3\right)\right] .
$$

With $k=m\left(m^{2}+1\right)$,

$$
k-K_{2}=\frac{\left(m^{2}+1\right)}{3}\left[2 m-\sqrt{ }\left(m^{2}-3\right)\right]>0
$$

and $k$ is too large for a $C 3$ solution to be possible.

Another possibility is to see what happens when $k$ is kept constant and $m$ is allowed to vary from $-\infty$ to $+\infty$. If we take $0 \leqslant y_{0} \leqslant 2 k$, then equation (1.1) gives a mapping into a fixed interval $[0,2 k]$ for all values of the parameter $m$. 
However, the behaviour of the solution depends on the value of $k$. To illustrate this we can start with $k=0.5$, which gives $F\left(y_{n}\right)=1 /\left\{1+\left(y_{n}-m\right)^{2}\right\}$. In this case there is a single equilibrium solution for each $m$ and the equilibrium solution is stable. To see this, consider the graph of $y=F_{0}(x)=1 /\left(1+x^{2}\right)$. This graph is symmetrical about $x=0$ and the maximum slope occurs at $x= \pm 1 / \sqrt{ } 3$ where the graph has a point of inflection. At the points of inflection the slope has magnitude $(3,8), 3=0.65$ and it follows thât the slope is less than 1 ai ail points. This means that $\left|F^{\prime}\left(y_{n}\right)\right|<1$ at all points and hence the line $y_{n+1}=y_{n}$ cannot intersect the graph of $y_{n+1}=F\left(y_{n}\right)$ in more than one point. Thus we get a single equilibrium point, say $Y$, with $\left|F^{\prime}(Y)\right|<1$, which ensures that the equilibrium is stable. This suggests that there should be no $C 2$ solutions and this can be checked by noting that $k^{*}=\left(m^{2}+1\right)\left(-m+\sqrt{ }\left(m^{2}+1\right)\right)$ has a minimum value which is greater than 0.5 . (Indeed $k=0.5$ was chosen to satisfy this condition is the first place.) We can infer that for $k=0.5$ there are no periodic solutions other than the equilibrium point.

The minimum value of $k^{*}$ occurs at $m=1 / \sqrt{ } 3$, where $k^{*}=(4 / 9) \sqrt{ } 3 \doteqdot 0.77$, so if we take $k=1$ we can be sure there will be $C 2$ solutions for some values of $m$. Now $k^{*}=1$ for $m=0$ and numerical work gives $k^{*}=1$ again for $m=m_{4}=$ 1.543689. Hence the $C 2$ solutions can only occur for $0<m<m_{4}$. From Paper $I$, the stability criterion for a $C 2$ solution is $S_{2}=\left(1+m^{2}\right)\left(1+m^{2}-2 k m\right) / k^{2}$, and this becomes $S_{2}=\left(1+m^{2}\right)(m-1)^{2}$ for $k=1$. With this expression for $S_{2}$, $S_{2}$ has a minimum value 0 at $m=1$ and $S_{2} \rightarrow 1$ as $m \rightarrow 0$ or $m \rightarrow m_{4}$. This checks that the $C 2$ solutions are stable, so no solutions with longer periods should occur. It was shown in Paper I that for equation (1.1) there could only be one equilibrium solution for $m<\sqrt{3}$ (for any choice of $k$ ) and when $k=1$ the discussion in Paper I can be used to show that there are three distinct equilibrium solutions only for $2<m<m_{5}$, where $m_{5}=2.134884$. For $m=2$ and $m=m_{5}$ there are double root solutions and for $m<2$ or $m>m_{5}$ there is only one real equilibrium solution. The equilibrium solution must be stable for $m<0$, for $m_{4}<m<2$ and for $m>m_{5}$, since $0<k<k^{*}$ in these intervals, and the equilibrium solution is unstable for $0<m<m_{4}$, where $k>k^{*}$. Where there are three equilibrium values, the smallest and the largest will be stable, with the intermediate one unstable. For $m>2$ we have $m>2 k$, so Coppel's conditions are satisfied [4, 2], and the iteration converges monotonically to an equilibrium value. Thus for $k=1$ and $m$ increasing we have an interval where there is a single stable equilibrium solution $(m<0)$, then an interval where there is a stable $C 2$ solution $\left(0<m<m_{4}\right)$, then another interval with a single stable equilibrium solution $\left(m_{4}<m<2\right)$, then an interval with one unstable and two stable equilibrium solutions $\left(2<m<m_{5}\right)$ and finally another interval with a single stable equilibrium solution $\left(m>m_{5}\right)$. This is a long way from the standard 
picture of period-doubling sequences and much closer to the "remerging Feigenbaum trees" described by Bier and Bountis [1].

If we take a slightly larger value for $k$, say $k=1.3$, chosen so that $k$ is greater than the minimum value of $k_{5}$, we can have stable $C 1$ solutions giving way to stable $C 2$ solutions as $m$ increases, then an interval where the $C 2$ solutions are unstable but stable $C 4$ solutions occur. As $m$ increases still further, the $C 4$ solutions become complex but there are stable $C 2$ solutions (over a shorter interval than before) before these $C 2$ solutions disappear and are replaced by $C 1$ solutions.

For $k=1.35$, the pattern is similar except that there are now two $m$-intervals where stable $C 4$ solutions occur. Between these two intervals there is a gap where $C 4$ solutions exist but are unstable and presumably stable $C 8$ solutions occupy at least part of this gap.

If we move to an appreciably larger value of $k$, say $k=5$, and keep $k$ fixed while $m$ increases from $-\infty$ to $+\infty$, the changes become more complex, and cannot be discussed in full with the equations available in this paper and in Paper I. We can introduce the notation that

$$
\begin{aligned}
& M_{1}=\left(\text { value of } m \text { for which } k_{1}=5\right) \quad(i=5,6,7,8) \text {, } \\
& M_{i}^{*}=\left(\text { value of } m \text { for which } k_{i}^{*}=5\right) \quad(i=5,6,7,8) \text {, } \\
& M_{-}^{*}, M_{+}^{*}=\left(\text { values of } m \text { for which } k^{*}=5\right) \text {, } \\
& M_{j}=\left(\text { value of } m \text { for which } K_{j}=5\right) \quad(j=1,2) \text {, } \\
& M_{j}^{*}=\left(\text { value of } m \text { for which } K_{j}^{*}=5\right) \quad(j=1,2),
\end{aligned}
$$

and we can take $M_{-}^{*}<0, M_{+}^{*}>0$ to distinguish these two values. The various $M$ and $M^{*}$ values are now critical values of $m$ in the sense that there will be

(i) stable $C 1$ solutions for $m<M_{-}^{*}$ and $m>M_{+}^{*}$,

(ii) stable $C 2$ solutions in $\left(M_{-}^{*}, M_{6}\right)$ and $\left(M_{5}, M_{+}^{*}\right)$,

(iii) stable $C 4$ solutions in $\left(M_{6}, M_{6}^{*}\right),\left(M_{8}, M_{8}^{*}\right),\left(M_{7}^{*}, M_{7}\right)$ and $\left(M_{5}^{*}, M_{5}\right)$,

(iv) stable $C 3$ solutions in $\left(M_{2}, M_{2}^{*}\right)$ and $\left(M_{1}^{*}, M_{1}\right)$.

Numerically, these critical values are:

$$
\begin{aligned}
& M_{\sim}^{*}=-1.020498, \quad M_{6}=1.239384, \quad M_{6}^{*}=1.488224, \quad M_{2}=2.00 \text {, } \\
& M_{2}^{*}=2.383399, \quad M_{8}=2.807304, \quad M_{8}^{*}=2.941606, \quad M_{7}^{*}=9.798271 \text {, } \\
& M_{7}=9.798392, \quad M_{1}^{*}=9.817682, \quad M_{1}=9.819772, \quad M_{5}^{*}=9.860592 \text {, } \\
& M_{5}=9.873000, \quad M_{+}^{*}=9.924557 \text {. }
\end{aligned}
$$

There are obviously large gaps in the information available but it looks as if we have period-doubling at first (as $m$ increases) and solutions of different period appear in the usual sequence until we have stable $C 3$ solutions in $\left(M_{2}, M_{2}^{*}\right)$. 
Then there is a large interval in which solutions of all periods are present, mostly unstable but with occasional small intervals of stability. Close to $m=10$ there is a smaller interval in which the $C 3$ solutions are stable and then the whole process reverses itself very rapidly as $m$ approaches $2 k$. The $C 3$ solutions disappear at $m=M_{1}$, the $C 4$ solutions at $m=M_{5}$ and the $C 2$ solutions at $m=M_{+}^{*}$, leaving the equilibrium solutions as the only periodic solutions for $m>M_{+}^{*}$.

Similar behaviour occurs for $k=10$. where the usual process of hifurcation reverses itself very rapidly as $m \rightarrow 2 k=20$. Indeed some preliminary runs indicate that this complete reversal takes place for smaller values of $k$, down to about $k=1.675$. For $k=1.674$ there are $C 3$ solutions, with a single interval of stability and no $C 3$ solutions outside this interval. Values of the stability criterion down to -0.997 were obtained but they appeared to remain just above -1 . For $k=1.675$, the situation was almost the same except that the minimum value for the stability criterion was about -1.025 and in consequence there were two separate intervals of stability for these $C 3$ solutions. The two intervals of stability were of comparable length. It is only as $k$ becomes larger that the first interval of stability for the $C 3 \mathrm{k}$ solutions becomes much larger than the second one.

There is obviously plenty of scope for examining other relationships between $m$ and $k$, but the examples above show how wide a range of behaviour is possible for one-parameter mappings.

In concluding, I should like to thank $\mathrm{Mr}$ W. A. Coppel for drawing my attention to the paper by Bier and Bountis [1], which encouraged me to extend the work on solutions with $k$ constant. The investigation was carried out as a Visiting Fellow in the Research School of Physical Sciences, Australian National University and I am grateful to the Department of Theoretical Physics for the facilities it has provided.

\section{References}

[1] M. Bier and T. C. Bountis, "Remerging trees in dynamical systems", Phys. Letters 104A (1984), 239-244.

[2] A. Brown, "A nonlinear difference equation with two parameters", J. Austral. Math. Soc. Ser. B 26 (1985), 430-451.

[3] P. Collet and J. P. Eckmann, lterated maps on the interval as dynamical systems (Birkhäuser, Boston, Mass., 1980).

[4] W. A. Coppel, "The solution of cubic equations by iteration", Z. Angew. Math. Phys. 9a (1958), 380-383.

[5] P. J. Holmes, "Bifurcation sequences in horseshoe maps: infinitely many routes to chaos", Phys. Letters 104A (1984), 299-302.

[6] G. L. Oppo and A. Politi, "Collisions of Feigenbaum cascades", Phys. Rev. 30A (1984), 435-441. 